\section{AFM in Biology - A Case Study}

The form and fibriliar organization of collagen is a key factor in determining the properties of many animal tissues. We have developed a protocol for nucleating collagen fibers and examining them in the AFM?

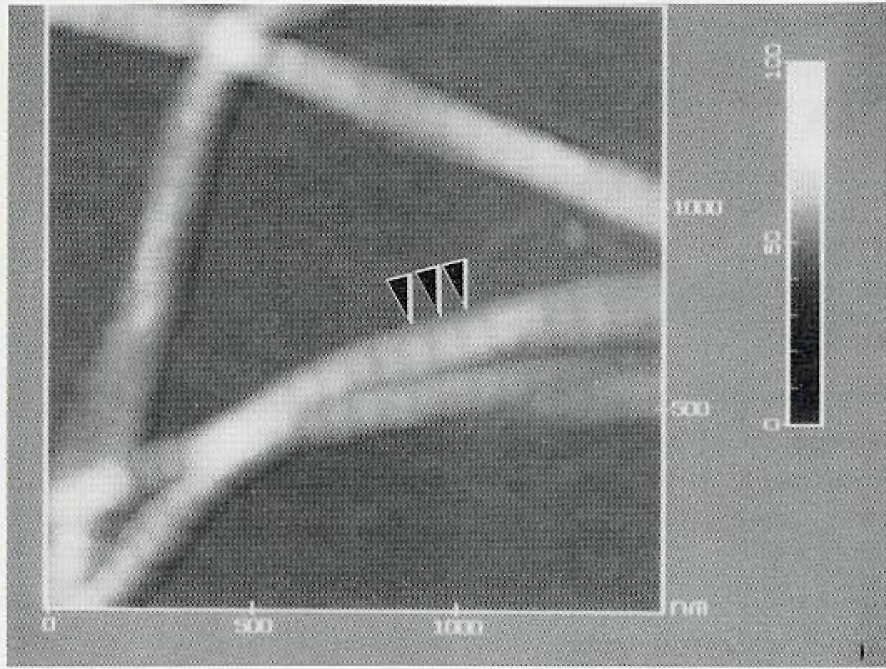

The above shows well-formed Type I collagen fibers which have a characteristic thickness variation at 70-nm intervals; this image was made in air with the Digital Instruments contact AFM.

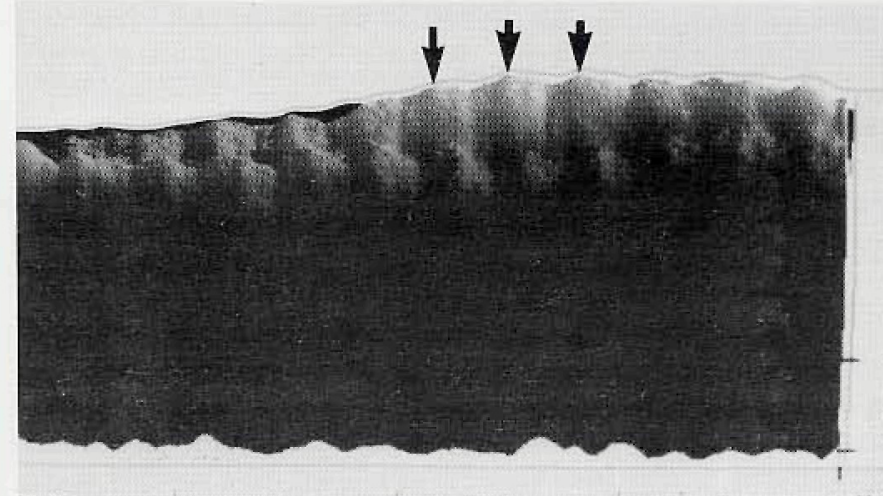

0.250

0.500

0.750

NM

The above shows similar fibers as previously but imaged by the new Digital Instruments non-contact AFM, operating in "Tapping Mode"3. This mode provides higher resolution because the probe touches the soft sample much more gently. For the first time with AFM, we are able to see not only the major bands (arrows), but also subbands in a repeating pattern of "wide valley-bump-bump". The asymmetry of this pattern agrees with the banding pattern seen in TEM with negative staining ${ }^{4}$ and this allows us to assign the left-hand side of this fiber as the C-terminus. This work shows that AFM examination (in both contact and non-contact modes) is a practical method for high resolution study of soft polymeric materials directly, without fixation, critical point drying, staining, or replica preparation.

\section{References}

1. D.A. Chernoff and H. Windischmann, "Atomic force microscope imaging for process characterization in diamond film desposition", J. Vac. Sci. Technol. A 102126 (1992).

2. E.A.G. Chernoff and D.A. Chernoff, "Atomic force microscope images of collagen fibers", J. Vac. Sci. Technol. A 10596 (1992)

3. E.A.G. Chernoff, D.A. Chernoff and Kkk. Kjoller, "Cpontact and Non-Contact atomic force microscopy of type I Collegen". American Society for Cell Biology. Annual Meeting 1992, abstract \#1316.

4. KA. Piez, Encyclopedia of Polymer Science and Engineering (Wiley, New York, 1985), Vol. 3, pp. 705-706

\section{AFM in Materials Science - A Case Study}

The control of thin film morphology is important to many applications, e.g. in microelectronics and optics. We have applied the AFM to characterize the effects of process parameters in diamond film deposition ${ }^{1}$.

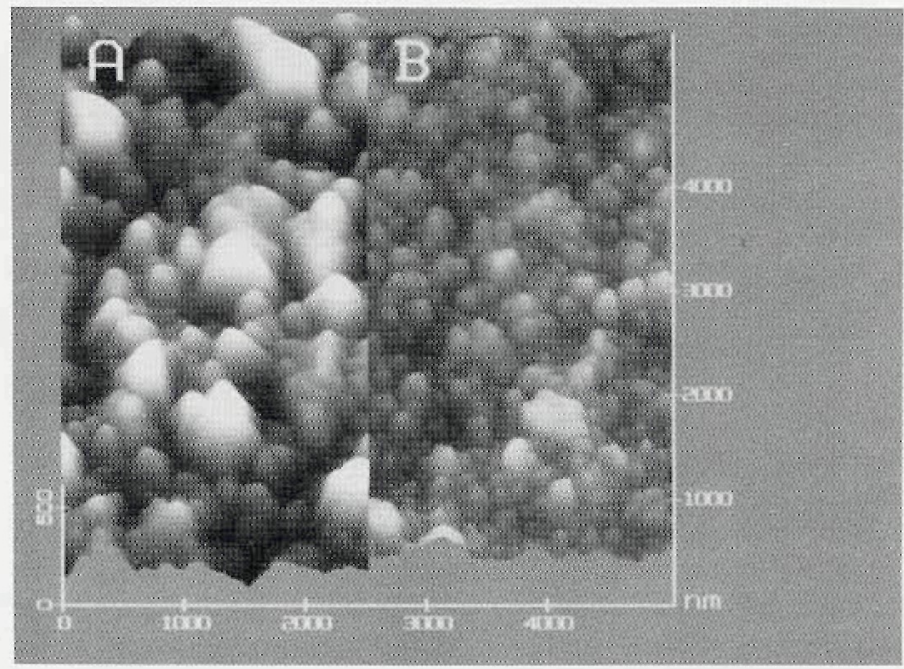

The above compares the grain size and surface roughness of diamond films produced by microwave plasma chemical vapor deposition. (A) has large grains and a rough surface (Rrms $=80 \mathrm{~nm})$. (B) has small grains and a smother surface (Rrms $=25 \mathrm{~nm}$ ) more suited for the intended application as a mask substrate in $x$-ray lithography. The ability to correlate roughness directly with morphology in a series of samples confirmed the relationship of grain size to methane concentration in the feed gas. As shown here, the AFM images grains distinctly in thin films as produced; no etching is reeded.

\section{A New Feature: History and Profiles of Companies Involved in Microscopy}

Beginning with this issue, Microscopy Today with carry articles covering the founding, early history and growth of companies that are engaged in producing or providing instrumentation, accessories, materiais or services for microscopy. Although we hope that many of the articles will be written by the founders themselves; corporate officers or long-time employees to provide a personal inside view of the companies, we must reserve the right to refuse articles or edit articles that are toolong or overly promotional.

\section{E.A. Fischione Instruments, Inc. Export, PA}

The thriving E.A. Fischione Instruments company began in the mid 60's, while Eugene Fischione was employed as a research machinist by US Steel in its Fundamental Research Laboratory in Monroeville, PA (Pittsburgh suburb). The Bain Lab was in its heyday with a large staff of energetic and creative scientists with money to spend on equipment. The highly competent "Gene" Fischione was much in demand to make the special devices that everybody wanted and the old refrain "the line forms on the left, dear" describes the situation rather well. With three new TEMs in the lab and a 1 million volt version on the way, many of Gene's projects involved EM devices and specimen preparation equipment. Frequently, visitors to the Bail Lab saw the custom-made devices and craved them on sight as they recognized the close connection between good specimen preparation the remarkable productivity of the lab 